\title{
Asplenia in children with congenital heart disease as a cause of poor outcome
}

\author{
SEMIHA BAHCECI ERDEM ${ }^{l}$, FERAH GENEL ${ }^{l}$, BARIS ERDUR ${ }^{l}$, ERHAN OZBEK ${ }^{1}$, \\ NESRIN GULEZ , TIMUR MESE
}

${ }^{1}$ Department of Pediatric Allergy and Immunology Department, Dr. Behcet Uz Children Training and Research Hospital, İzmir, Turkey ${ }^{2}$ Department of Pediatric Cardiology, Dr. Behcet Uz Children Training and Research Hospital, İzmir, Turkey

\begin{abstract}
The absence of a spleen is a well-known risk factor for severe bacterial infections, especially due to encapsulated bacteria. Congenital asplenia can be part of multiple congenital abnormalities as in heterotaxy including Ivemark syndrome with congenital anomalies of the heart or great vessels, or it can be isolated, which is extremely rare. In these cases, asplenia is an important factor effecting mortality. In this report, the clinical courses of five children with asplenia and concomitant minor or complex cardiac anomalies are presented. The ages of the children ranged between 1.5 and 17 months at the time of diagnosis. All of the cases had had a history of hospitalisation for infectious diseases before the diagnosis. The patient who was diagnosed at 17 months old had a history pneumonia, urinary tract infection, and bacterial meningitis beginning at five months old. Three children had complex cardiac anomalies, one child had ventricular septal defect, and one child had atrial septal defect. Howell-Jolly bodies were determined in peripheral blood smear in all of the patients. The diagnoses of asplenia were confirmed with spleen scintigraphy. One of the patients with complex cardiac anomalies died a short time after diagnosis, because of cardiac failure. The rest of the four patients were vaccinated for encapsulated bacteria and were taken under antibiotic prophylaxis. These children did not need hospitalisation for infectious diseases during the follow-up period (5-40 months). In asplenic children, early diagnosis, antibiotic prophylaxis, and immunisation for encapsulated bacteria can decrease the risk of morbidity and mortality.
\end{abstract}

Key words: asplenia, child, cardiac anomalies, heterotaxy syndrome, infection, immunisation.

(Centr Eur J Immunol 2015; 40 (2): 266-269)

\section{Introduction}

Congenital asplenia may occur in isolation. However, it is usually part of a syndrome and is accompanied by abnormalities in other organ systems, especially cardiac abnormalities. Heterotaxy syndrome with asplenia and right atrial isomerism is the most frequent one of these syndromes, occurring approximately in 1 in 10,000-40,000 births. Both isolated and syndromic asplenia are associated with increased incidence of life-threatening invasive infections. In cases with heterotaxy, heart disease is the most common cause of mortality, especially in the first year of life, but fatal infections have also been reported. In these patients, asplenia is an important factor effecting mortality. Early diagnosis and treatment of asplenia in children with cardiac malformations can decrease the additional risk of mortality and morbidity. In this report we present the clinical courses of five children with asplenia and concomitant minor or complex cardiac anomalies.

\section{Case report}

The ages of the children ranged between 1.5 and 17 months at the time of diagnosis. Three of the patients were male and two of them were female. All of the cases had a history of hospitalisation for infectious diseases before the diagnosis. The patient who was diagnosed at 17 months old had a history of pneumonia, urinary tract infection, and bacterial meningitis beginning at five months old. Three children had complex cardiac anomalies, one child had ventricular septal defect, and one child had atrial septal defect. One of the children (case 4) with complex cardiac defects had additional anomalies such as unilateral renal agenesia and syndactyly. Howell-Jolly bodies were determined in peripheral blood smear in all of the patients. The diagnoses of asplenia were confirmed with spleen scintigraphy. One of the patients with complex cardiac anomalies (dextrocardia, total anomalous pulmonary venous return, right atrial isomerism, atrioventricular septal defect, transposition of great arteries, pulmonary stenosis)

Correspondence: Semiha Bahceci Erdem, Department of Pediatric Allergy and Immunology Department, Dr. Behcet Uz Children Training and Research Hospital, 35260 İzmir, Turkey, e-mail: semihabahceci@hotmail.com 


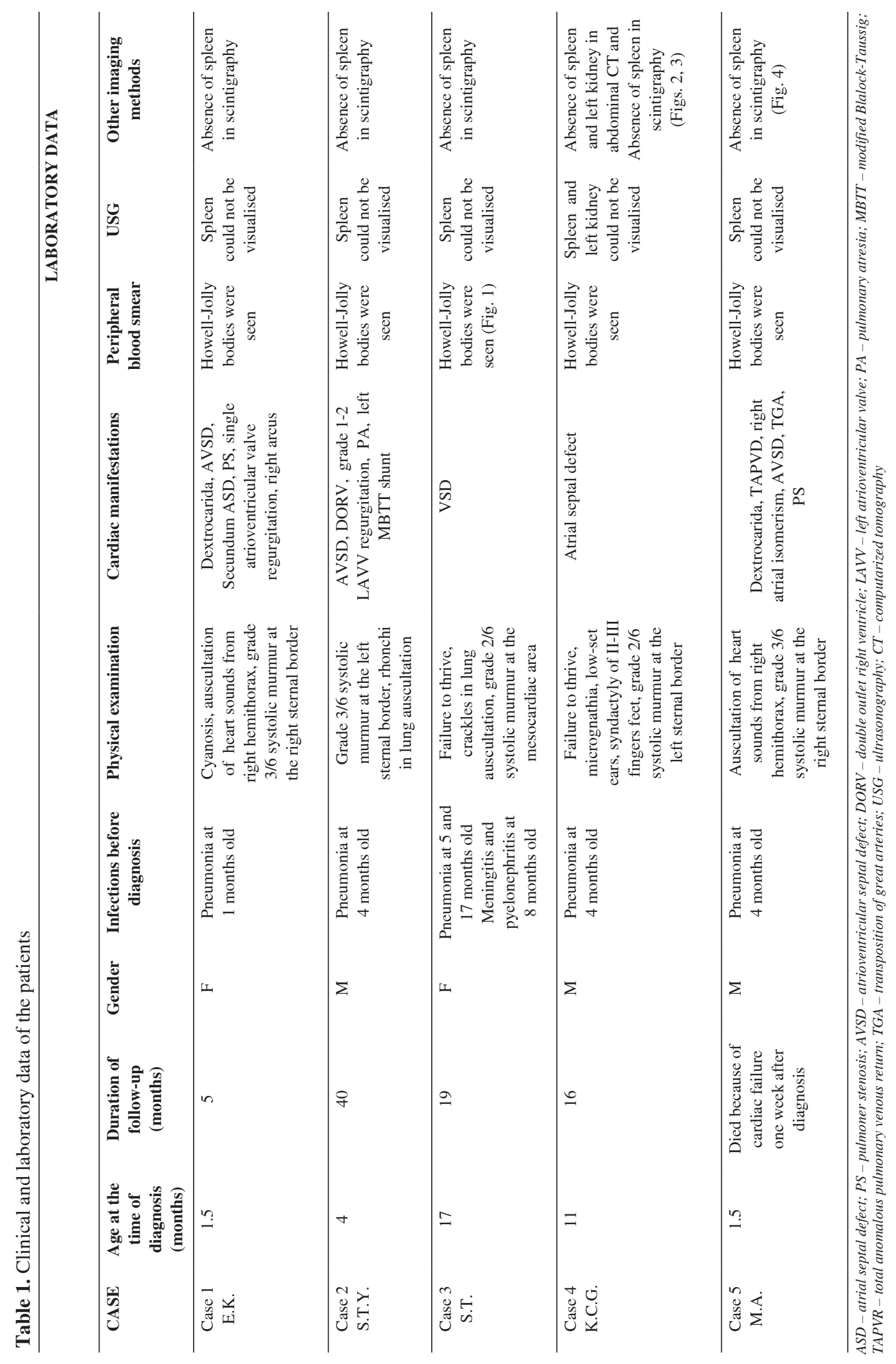


died one week after diagnosis, because of cardiac failure. The remaining four patients were vaccinated for encapsulated bacteria and were taken under antibiotic prophylaxis. These children did not need hospitalisation for infectious diseases during the follow-up period (5-40 months). Clinical and laboratory data of the patients are given in Table 1.

\section{Discussion}

Asplenia may be acquired (after trauma and surgery), functional (sickle cell anaemia), or congenital. Congenital asplenia is a rare but life-threatening condition. It may arise as a part of situs abnormalities or it may be isolated. Isolated congenital asplenia is a very rare condition, and the mode of inheritance is usually autosomal dominant. Some sporadic cases have also been reported [1]. Heterotaxy syndrome-asplenia and polysplenia is more common. Patients with heterotaxy syndrome often present with congenital heart disease but $5-10 \%$ of the patients do not have major cardiac malformations. It is difficult to diagnose when an asplenic patient does not have any concomitant anomalies, and diagnosed cases post mortem have been reported [2, 3]. In heterotaxy syndromes mutations in connexin 43 and ZIC3 genes have been demonstrated. In isolated congenital asplenia, ribosomal protein SA haploinsufficiency has been detected [4]. In our series of five patients, three cases had complex cardiac malformations, one patient had ventricular septal defect, and one patient had atrial septal defect.

The spleen is an important organ in phagocytosis of encapsulated bacteria and immunoglobulin production against these agents [2]. In asplenic patients, the risk of death from septicaemia is 200 times higher than in patients with a spleen. The bacteria causing septicaemia are predominantly pneumococci (50-80\% of identified infections) and meningococci, Haemophilus sp., and other capsulated

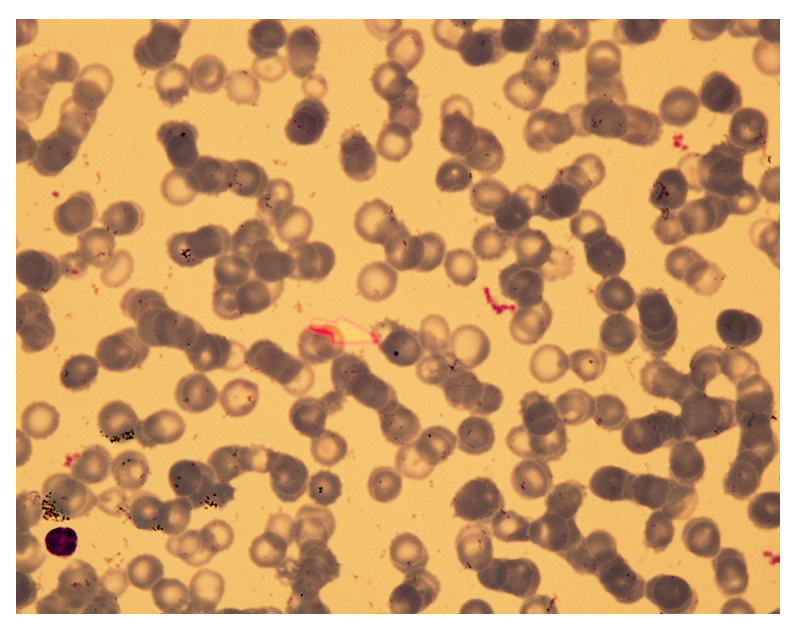

Fig. 1. Howell-Jolly bodies in the peripheral blood smear (case 3) bacteria $[2,5,6]$. In the literature, the youngest congenital asplenic patient was diagnosed with meningitis by Escherichia coli occurred for days after birth [2]. Congenital asplenia detected in a 60-year-old woman with septicaemia by streptococcus pneumonia was also reported [7]. However, some patients may be asymptomatic. These data show the variations in the clinical course among patients, even in members of the same family [1]. Asplenia may be a cause of sudden, unexpected death. Kanthan et al. presented a previously healthy four-year-old girl with sudden death who had sporadic, isolated congenital asplenia complicated by pneumococcus sepsis and bilateral adrenal haemorrhage diagnosed at autopsy [8]. In a French multicentre study 293 children with sudden death were investigated at autopsy. Four cases of asplenia were identified, three of which were with syndromic asplenia and one of which was with isolated congenital asplenia [9]. In asplenic patients, meningitis, septicaemia, and purpura fulminans are encountered as severe invasive infections. The rate of invasive bacterial infections, mortality, and major sequelae have been reported as $69 \%, 64 \%$, and $7 \%$, respectively [2]. Heterotaxy with asplenia leads to a high morbidity and mortality [10]. Mikate et al. evaluated 8923 cases operated due to congenital heart disease. Asplenia has been shown to be one of the postoperative risk factors influencing mortality when operated due to congenital heart disease, while with heterotaxy, morbidity and mortality increases [11]. In all of our patients there was a history of hospitalisation because of lower respiratory infection before the diagnosis of asplenia. One patient had a history of urinary tract infection and acute bacterial meningitis additionally.

The presence of Howell-Jolly bodies in peripheral blood smear is an important sign of congenital or functional asplenia [7]. Therefore, careful examination of peripheral blood smear is important in children with congenital heart disease. When Howell-Jolly bodies are present in peripheral blood smear, the diagnosis of asplenia must be confirmed by ultrasonography, computerised tomography, magnetic resonance imaging, or spleen scintigraphy [9]. In all of our cases, the spleen could not be imaged by ultrasonography. The diagnosis of asplenia was confirmed by computerised tomography in one of the cases, and by spleen scintigraphy in four of the cases.

By antibiotic prophylaxis and immunisation for pneumococcal infections and haemophilus influenzae type $b$, protection from invasive infections is possible [2, 12, 13]. Although conjugated vaccines increase protection from pneumococcal infections, $19 \%$ of the patients are prone to infections by serotypes out of the content of heptavalent pneumococcal vaccine. Moreover, vaccine induced immunity may be defective in asplenic patients. Therefore, fever of unknown origin must be evaluated carefully in asplenic patients $[2,5,13]$. The four of our cases were taken under antibiotic prophylaxis and were vaccinated for encapsulated bacteria. These children did not necessitate 


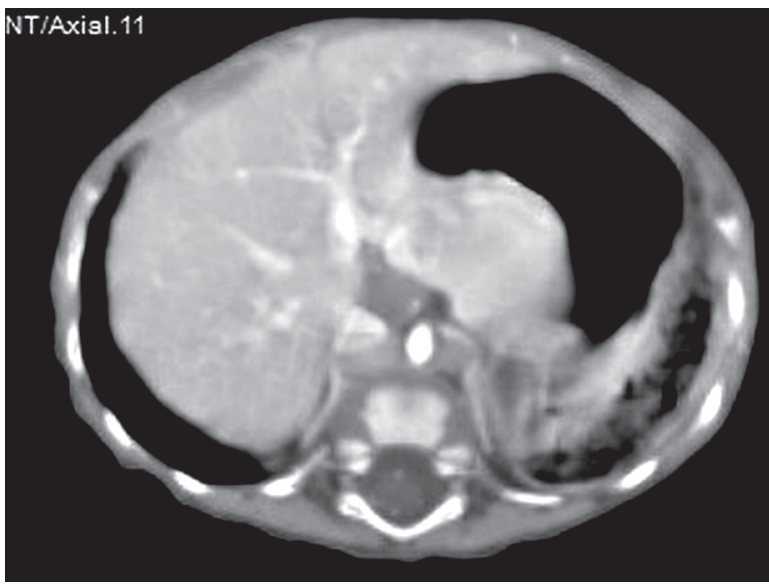

Fig. 2. Absence of spleen in abdominal computerised tomography (case 4)

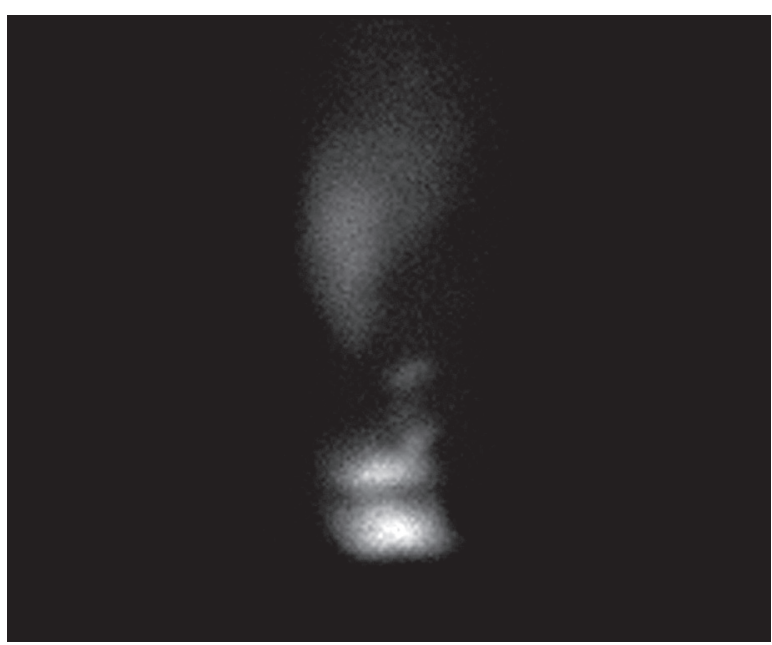

Fig. 4. Absence of spleen in scintigraphy (case 5)

hospitalization for infection diseases during the follow-up period (5-40 months). One of our cases, who had complex cardiac malformations, was lost owing to heart failure on the seventh day after diagnosis.

\section{Conclusions}

Asplenia can be accompanied by major or minor cardiac anomalies. The existence of Howel-Jolly bodies in peripheral blood smear is useful for determining asplenia in patients with cardiac anomalies. In asplenic children, early diagnosis, antibiotic prophylaxis, and immunisation for encapsulated bacteria, can decrease the risk of morbidity and mortality due to invasive infections.

The authors declare no conflict of interest.

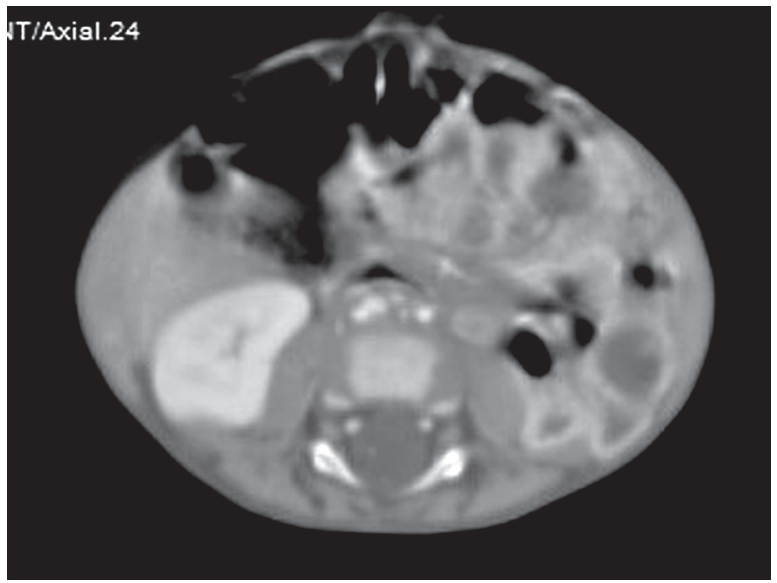

Fig. 3. Absence of left kidney in abdominal computerised tomography (case 4)

\section{References}

1. Ahmed SA, Zengeya S, Kini U, Pollard AJ (2010): Familial Isolated congenital asplenia: case report and literature review. Eur J Pediatr 163: 315-318.

2. Uchida Y, Matsubaro K, Wada T, et al. (2012): Bacteriel meningitis by three different pathogens in an isolated asplenic child. J Infect Chemother 18: 576-578.

3. Celik M, Celik T, Iyisoy A, Guler A (2011): An unusual combination of congenital anomalies in an adult patient: patent ductus arteriosus, Kommerell's diverticulum with aberrant right subclavian artery, and heterotaxy syndrome. Hellenic J Cardiol 52: 469-472.

4. Bolze A, Mahlaoui N, Byun M, et al. (2013): Ribosomal protein SA haploinsufficiency in humans with isolated congenital asplenia. Science 340: 976-978.

5. Schutze GE, Mason EO Jr, Barson WJ, et al. (2002): Invasive pneumococcal infections in children with asplenia. Pediatr Infect Dis J 21: 278-282.

6. Eytout J, Tournilhac O, Laurichesse H (2003): Asplenia and hyposplenism. Presse Med 32 (28 suppl): S5-S9.

7. Germing U, Perings C, Steiner S, et al. (1999): Congenital asplenia detected in a 60 year old patient with septicemia. Aul C.Eur J Med Res 4: 283-285.

8. Kanthan R, Moyana T, Nyssen J (1999): Asplenia as a cause of sudden unexpected death in childhood. Am J Forensic Med Pathol 20: 57-59.

9. Mahlaoui N, Minard-Colin V, Picard C, et al. (2011): Isolated congenital asplenia: a French nationwide retrospective survey of 20 cases. J Pediatr 158: 142-148.

10. Burton EC, Olson M, Rooper L (2014): Defects in laterality with emphasis on heterotaxy syndromes with asplenia and polysplenia: an autopsy case series at a single institution. Pediatr Dev Pathol 17: 250-264.

11. Miyata H, Murakami A, Tomotaki A, et al. (2014): Predictors of 90-day mortality after congenital heart surgery: the first report of risk models from a Japanese database. J Thorac Cardiovasc Surg 148: 2201-2206.

12.Prendille TW, Barton LL, Thompson WR, et al. (2010): Heterotaxy Syndrome: Defining Cotemporary Disease Trends. Pediatr Cardiol 31: 1052-1058.

13. Waghorn DJ (2001): Overwhelming infection in asplenic patients: current best practice preventive measures are not followed. J Clin Pathol 54: 214-218. 